Ute Wölfle ${ }^{a}$

Christoph M. Schempp ${ }^{\text {a }}$

\title{
Flavonoide als vielseitige Wirkstoffe für die Haut - Fokus Luteolin
}

keit mit körpereigenem steroidalem Östrogen können Isoflavonoide an den Östrogenrezeptor binden. Generell ist die Bindung der Phytoöstrogene allerdings schwächer als diejenige der steroidalen Östrogene. Je nachdem wie hoch die Konzentration von gleichzeitig vorhandenen steroidalen Östrogenen ist, wirken sie somit als Östrogene oder Antiöstrogene [2].

\section{Pharmakologie von Luteolin}

Unter den Flavonoiden gehört Luteolin zu den antioxidativ wirksamsten Polyphenolen im Pflanzenreich [3]. Luteolin kommt z.B. in der Färberpflanze Reseda luteola (Färberwau, Gilbkraut) in grösseren Mengen vor. Doch auch Erdnussschalen, Mandarinenschalen oder Chrysanthemen enthalten bedeutende Mengen an Luteolin. Luteolin konnte auch in Gewürzpflanzen wie dem Thymian und der Petersilie nachgewiesen werden, doch die Aufnahme von Luteolin durch die Nahrung ist sehr gering $(<1 \mathrm{mg} / \mathrm{Tag})$. Im Fall von R. luteola befindet sich der Hauptteil der Flavonoide in den Samenkapseln. $R$. luteola ist eine zweijährige Pflanze, die auf kalk- und kieshaltigen Böden wächst und ursprünglich aus Südeuropa stammt (Abb. 1).

Anhand von spektrophotometrischen Messungen mit 1\% (v/v) Luteolin konnte gezeigt werden, dass Luteolin UVB- und UVA-Licht absorbieren kann. Die UV-Transmission unterhalb von $370 \mathrm{~nm}$ beträgt weniger als $10 \%$; d.h., äusserlich aufgetragenes Luteolin kann den Eintritt von Photonen in die Haut in einem ersten
Schutzmechanismus zu einem Grossteil verhindern [4]. Des Weiteren wirkt Luteolin antientzündlich und neutralisiert reaktive Sauerstoffspezies (ROS), die z.B. durch Übertragung von elektromagnetischer Energie des UV-Lichts auf molekularen Sauerstoff entstehen [5]. Ausserdem vermindert Luteolin die Bildung von UVB-verursachten DNA-Schäden. Dadurch kann eine strahlungsbedingte Anhäufung von Mutationen, z.B. im Tumorsuppressorgen $\mathrm{p} 53$, verhindert werden. UV-spezifische Mutationen im p53Gen begünstigen die Entstehung einer präkanzerösen aktinischen Keratose, die eine Vorstufe des Plattenepithelkarzinoms darstellt [6]. Deshalb eignet sich äusserlich angewendetes Luteolin zur Prävention von aktinischen Keratosen und hellem Hautkrebs.

\section{Luteolin als Wirkstoff für die Haut}

Luteolin hemmt in Keratinozyten auch die UV-strahlungsbedingte Bildung und Aktivierung von MatrixMetalloproteinasen. Diese Enzyme zerstören in der Haut Kollagen und Elastin. Werden diese Schäden fehlerhaft repariert, kommt es mit der Zeit $\mathrm{zu}$ einem faltigen Erscheinungsbild der Haut, was als Lichtalterung oder Photoaging bezeichnet wird [7]. Auf diese Weise kann äusserlich angewendetes Luteolin das durch UV-Strahlung ausgelöste Photoaging der Haut verringern.

Nach neuesten Erkenntnissen spielen ROS auch bei der Entstehung irritativer und allergischer Kontaktekze- 
Abb. 1. a Strukturformel des PhenylchromanGerüstes sowie des Luteolins und $\mathbf{b}$ Reseda luteola (Foto: Margitta Paprotka-Kühne).

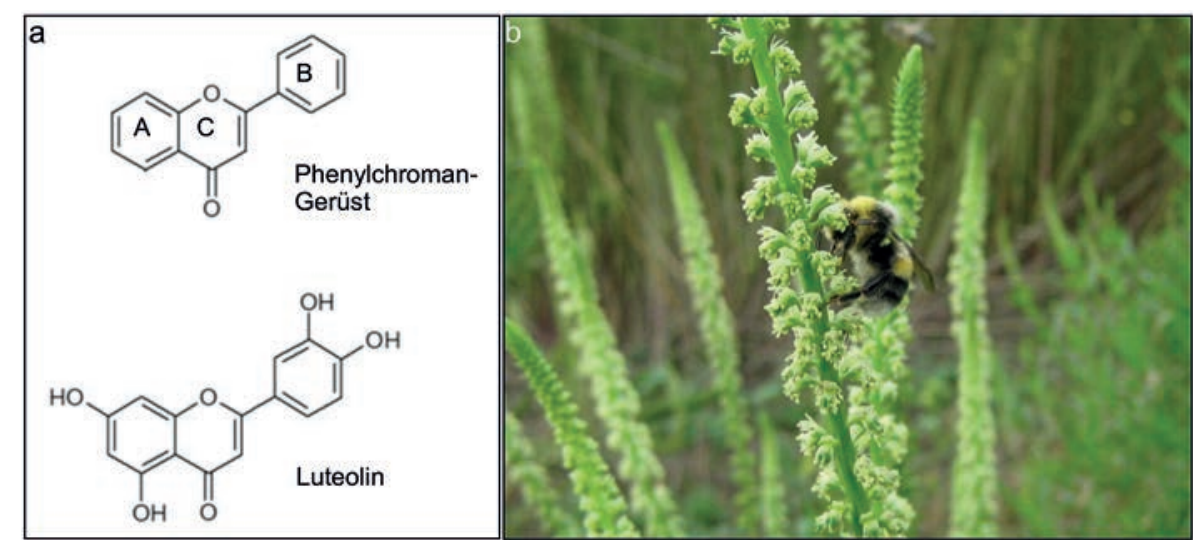

me eine Rolle [8]. Eine Antioxidantiencreme mit Luteolin schützt z.B. vor Hautirritationen, die durch häufiges Waschen und wasserlösliche Irritanzien (verdünnte Säuren und Laugen, Alkohol, Spül- und Waschmittel sowie Desinfektionsmittel) ausgelöst werden. Dieser Effekt konnte in einem standardisiert durchgeführten wiederholten Waschtest mit der Lauge Natriumlaurylsulfat an 25 gesunden Probanden verdeutlicht werden. Die Antioxidantiencreme verringerte signifikant die Rötung sowie den transepidermalen Wasserverlust der irritierten Haut und verbesserte die Hydratation der Hornschicht [9]. Im Gegensatz zum irritativen Kontaktekzem ist das allergische Kontaktekzem eine T-Zell-vermittelte entzündliche Hautkrankheit. Im Zuge einer Kontaktallergen-induzierten Hautentzündung werden dendritische
Zellen aktiviert, wandern von der Haut in hautdrainierende Lymphknoten und präsentieren die Kontaktallergenspezifischen zytotoxischen T-Zellen [8]. Der Kontakt von dendritischen Zellen mit dem Kontaktallergen führt ausserdem zur Bildung von ROS. Es konnte gezeigt werden, dass ROS auch Hyaluronidasen aktiviert, die schliesslich die Hyaluronsäure fragmentieren. Diese Hyaluronsäure-Fragmente können dann Toll-like-Rezeptoren (TLR) stimulieren [10], die normalerweise durch Bestandteile von bakteriellen Zellwänden und bakteriellen Toxinen aktiviert werden und zur Bildung von Entzündungsstoffen beitragen. ROS spielen in diesem System generell eine ganz zentrale Rolle. Luteolin wirkt allerdings nicht nur stark antioxidativ, sondern ist auch ein Inhibitor der Hyaluronidase, sodass weniger Hyaluron- säure abgebaut wird, die zur Aktivierung der TLR beitragen kann. Luteolin wirkt somit in mehrfacher Hinsicht der Entstehung einer Kontaktallergie entgegen. Deshalb ist denkbar, dass Luteolin präventiv die Auslösung einer Kontaktallergie bei entsprechenden Allergikern verhindern kann. Derzeit sind ca. 4000 Kontaktallergene bekannt, und 5-10\% der Bevölkerung sind gegen mindestens eine Substanz sensibilisiert [11]. Standardmässig wird die Kontaktallergie mit Glucocorticoiden und nichtsteroidalen antiinflammatorischen Medikamenten behandelt, die jedoch zum Teil schwerwiegende Nebenwirkungen haben. Der Einsatz von Antioxidantien, insbesondere von Luteolin, zur Prävention und Behandlung von allergischen Kontaktallergien stellt dabei einen neuen Ansatz dar.

\section{Literatur}

1 López-Lázaro M: Distribution and biological activities of the flavonoid luteolin. Mini Rev Med Chem 2009;9:31-59.

$\checkmark 2$ Ko K-P: Isoflavones: chemistry, analysis, functions and effects on health and cancer. Asian Pac J Cancer Prev 2014;15:7001-7010.

3 Seelinger G, Merfort I, Schempp CM: Anti-oxidant, anti-inflammatory and anti-allergic activities of luteolin. Planta Med 2008;74:1667-1677.

4 Wölfle U, Esser PR, Simon-Haarhaus B, Martin SF, Lademann J, Schempp CM: UVB-induced DNA damage, generation of reactive oxygen species, and inflammation are effectively attenuated by the flavonoid luteolin in vitro and in vivo. Free Radic Biol Med 2011;50: 1081-1093.
Dinkova-Kostova AT: Phytochemicals as protectors against ultraviolet radiation: versatility of effects and mechanisms. Planta Med 2008; 74:1548-1559.

6 Nishisgori C: Current concept of photocarcinogenesis. Photochem Photobiol Sci 2015;14: 1713-1721.

7 Wenk J, Brenneisen P, Meewes C, Wlaschek M, Peters T, Blaudschun R, Ma W, Kuhr L, Schneider L, Scharffetter-Kochanek K: UVinduced oxidative stress and photoaging. Curr Probl Dermatol 2001;29:83-94.

$\checkmark 8$ Martin SF, Esser PR, Weber FC, Jakob T, Freudenberg MA, Schmidt M, Goebeler M: Mechanisms of chemical-induced innate immunity in allergic contact dermatitis. Allergy 2011;66:1152-1163.
Schempp CM, Meinke MC, Lademann J, Ferrari $\mathrm{Y}$, Brecht $\mathrm{T}$, Gehring $\mathrm{W}$ : Topical antioxidants protect the skin from chemical-induced irritation in the repetitive washing test: a placebo-controlled, double-blind study. Contact Dermatitis 2012;67:234-237.

10 Gariboldi S, Palazzo M, Zanobbio L, Selleri S, Sommariva M, Sfondrini L, Cavicchini S, Balsari A, Rumio C: Low molecular weight hyaluronic acid increases the self-defense of skin epithelium by induction of beta-defensin 2 via TLR2 and TLR4. J Immunol 2008;81:21032110.

11 Martin SF: New concepts in cutaneous allergy. Contact Dermatitis 2015;72:2-10. 